\title{
Advanced Visualization of Experimental Data in Real Time Using LiveView3D
}

\author{
Richard J. Schwartz ${ }^{1}$ \\ Swales Aerospace, Hampton, VA, 23681 \\ Gary A. Fleming ${ }^{2}$ \\ NASA Langley Research Center, Hampton, VA, 23681
}

\begin{abstract}
LiveView3D is a software application that imports and displays a variety of wind tunnel derived data in an interactive virtual environment in real time. LiveView3D combines the use of streaming video fed into a three-dimensional virtual representation of the test configuration with networked communications to the test facility Data Acquisition System (DAS). This unified approach to real time data visualization provides a unique opportunity to comprehend very large sets of diverse forms of data in a real time situation, as well as in post-test analysis. This paper describes how LiveView3D has been implemented to visualize diverse forms of aerodynamic data gathered during wind tunnel experiments, most notably at the NASA Langley Research Center Unitary Plan Wind Tunnel (UPWT). Planned future developments of the LiveView3D system are also addressed.
\end{abstract}

\section{Introduction}

$\mathrm{D}^{2}$ ata Acquisition Systems (DAS) in modern wind tunnel facilities provide the ability to acquire vast quantities of data during wind tunnel tests. It is not unusual for a wind tunnel model to be outfitted with dozens to hundreds of surface pressure taps, electronic pressure sensors, and a six-component force balance. Video-based flow diagnostic techniques ${ }^{1}$ such as schlieren imaging and laser light sheet flow visualization are also commonly used. Thus the volume of data collected during a single day of moderately intensive wind tunnel testing can reach multiple gigabytes. This leads to the associated problem of having to process, analyze, and visualize all of this data in order to identify and extract the important parts that will yield knowledge about the phenomena under test. To aid this process, a software application called LiveView3D has been developed at NASA Langley Research Center (LaRC). LiveView3D has been designed to unify and display data of different types, such as scalar surface pressure measurements and schlieren video, in real time as the data are being acquired. The data are displayed in a spatially accurate, virtual representation of the test environment, which provides the researcher with the ability to view and consider the data with a global perspective. The real-time visualization of combined data sets in the virtual environment can lead to a greater empirical understanding about the test while it is happening. This is in stark contrast to traditional methods of data interrogation, which often involves the sequential consideration of data produced by independent instruments, generally occurring after the test campaign has been completed.

LiveView3D has been designed to co-exist with current wind tunnel DAS and advanced instrumentation. It is meant to provide the researcher with the latest information available from the various measurement techniques being utilized, and it does so by combining the datasets from each instrument into a virtual

\footnotetext{
${ }^{1}$ Senior Research Engineer, Measurement Sciences and Instrumentation, 18 Langley Blvd, NASA Langley Research Center, Hampton, VA 23681 / Mail Stop 493.

${ }^{2}$ Assistant Branch Head, Advanced Sensing and Optical Measurement Branch, 18 Langley Blvd, NASA Langley Research Center, Hampton, VA 23681 / Mail Stop 493.
} 
environment that closely mimics the actual test configuration. A key aspect of LiveView3D is that the user may interact with the virtual environment in real time, navigating through the three-dimensional space. This provides an intuitive way to identify trends and dependencies within very large data sets.

LiveView3D can also be utilized as a mechanism to determine if the instrumentation is working as expected. Many anomalies (such as a clogged pressure tap, for example) can be quickly identified through the real-time visualization. LiveView3D also provides the researcher with the ability to mentally correlate the response of multiple instrumentation systems to ensure they are behaving in a self-consistent manner. Most importantly, however, LiveView3D can provide the researcher with an empirical understanding of the physics occurring within the test in real time. This avails the possibility of altering the test plan to further explore unexpected observations or results, something that is often impossible to do if the data are analyzed after a test has been completed and the hardware is removed from the facility.

This report describes the current capabilities of LiveView3D and provides examples of how it has been utilized for real-time data visualization at NASA LaRC. Future developments planned for LiveView3D are also addressed. While this paper concentrates on describing the real time data visualization capabilities of LiveView3D, the program is also capable of reading and displaying archived data from a variety of formats. This allows LiveView3D to also be used for post-test data visualization.

\section{LiveView3D}

The LiveView3D data visualization system is based on a workstation-class PC-compatible computer using the Windows ${ }^{2} \mathrm{XP}$ operating system. The computer is currently equipped with a commercially available frame grabber capable of capturing video from industry standard RS-170 analog monochrome video cameras. The computer is also outfitted with a professional-grade graphics card with dual monitor outputs.

The LiveView3D software consists of two separate computer programs that work in unison; the LiveView3D Controller and the three-dimensional graphical visualization environment. The LiveView3D Controller is a custom computer program written in Microsoft Visual Basic ${ }^{3}$. The Controller provides a graphical user interface to allow the researcher to control various aspects of the data visualization, and to save and recall data streams for display. The three-dimensional graphical visualization environment is provided by AutoDesk 3ds $\mathrm{Max}^{4}$. 3ds Max is a CAD-like software package with advanced modeling, lighting, rendering, and animation toolsets for generating very high quality interactive visualizations. The LiveView3D Controller communicates with $3 \mathrm{ds}$ Max using the Microsoft ActiveX $\mathrm{X}^{5}$ protocol. This allows user input parameters from the Controller to be passed to custom-developed code within 3ds Max, whereby the user-requested changes to the visualization are implemented. Further hardware and software details are available in Reference 6.

LiveView3D has been used at several facilities to visualize streaming video. However, in order to fully exploit the capabilities of the LiveView3D software, data from the existing wind tunnel DAS is incorporated into the visualization. This was implemented at the NASA LaRC Unitary Plan Wind Tunnel (UPWT), whose DAS operates on a UNIX-based MODCOMP computer platform. The LiveView3D and UPWT DAS computers were interfaced via Ethernet and used TCP/IP for communications. This allowed a stream of up to 500 scalar parameters acquired and/or computed by the DAS to be transmitted and incorporated into the LiveView3D visualization.

For all of the examples discussed below, a pre-requisite is a three-dimensional Computer Aided Design (CAD) model of the test configuration. Typically, this consists of the merging of a detailed scale representation of the test facility with a three-dimensional CAD file of the wind tunnel model being tested. Facility models have historically been created in 3ds Max based on blueprints, photographs and measurements. Creation of a facility model generally takes a few weeks, including adding dynamic elements such as the motion of stings and model injection systems.

To complete the virtual environment, the test article is added to the facility. A three-dimensional CAD model, usually available from the model designer, is imported and scaled to match the facility. It is then linked to the sting mechanism, which will allow the model to move in the same way as the actual test 
hardware. Commercially available CAD file translators have made this a quick and relatively trivial part of the process.

\section{LiveView3D Operations}

The LiveView3D system has been used in conjunction with schlieren and laser light sheet flow visualization imagery in real time. It has also been applied to scalar values from surface pressure taps and six component force balances. The following sections will describe some of the tests that have utilized LiveView3D for real time data visualization.

\section{A. Flow Visualization via Live Video.}

One of the most unique capabilities of LiveView3D is the ability of the software to display live video in the interactive three-dimensional virtual environment in real time. This is accomplished using a commercially available video frame grabber installed in the LiveView3D computer. The LiveView3D system currently uses an RS-170 analog video frame grabber, but frame grabbers for other video transmission protocols could be easily substituted. Each digitized frame of video is converted into a bitmap and is subsequently mapped to a spatially-registered position within the virtual environment. The video update rate in the virtual environment is approximately 15 frames / second when using a 2003-era, dual-processor PCcompatible computer and a standard $30-\mathrm{Hz}$ video camera. The LiveView3D system can be independently utilized to display live video in a virtual environment without connecting to any other data sources or facility data acquisition system (figure 1). This makes the live video capability highly transportable and relatively easy to set up.

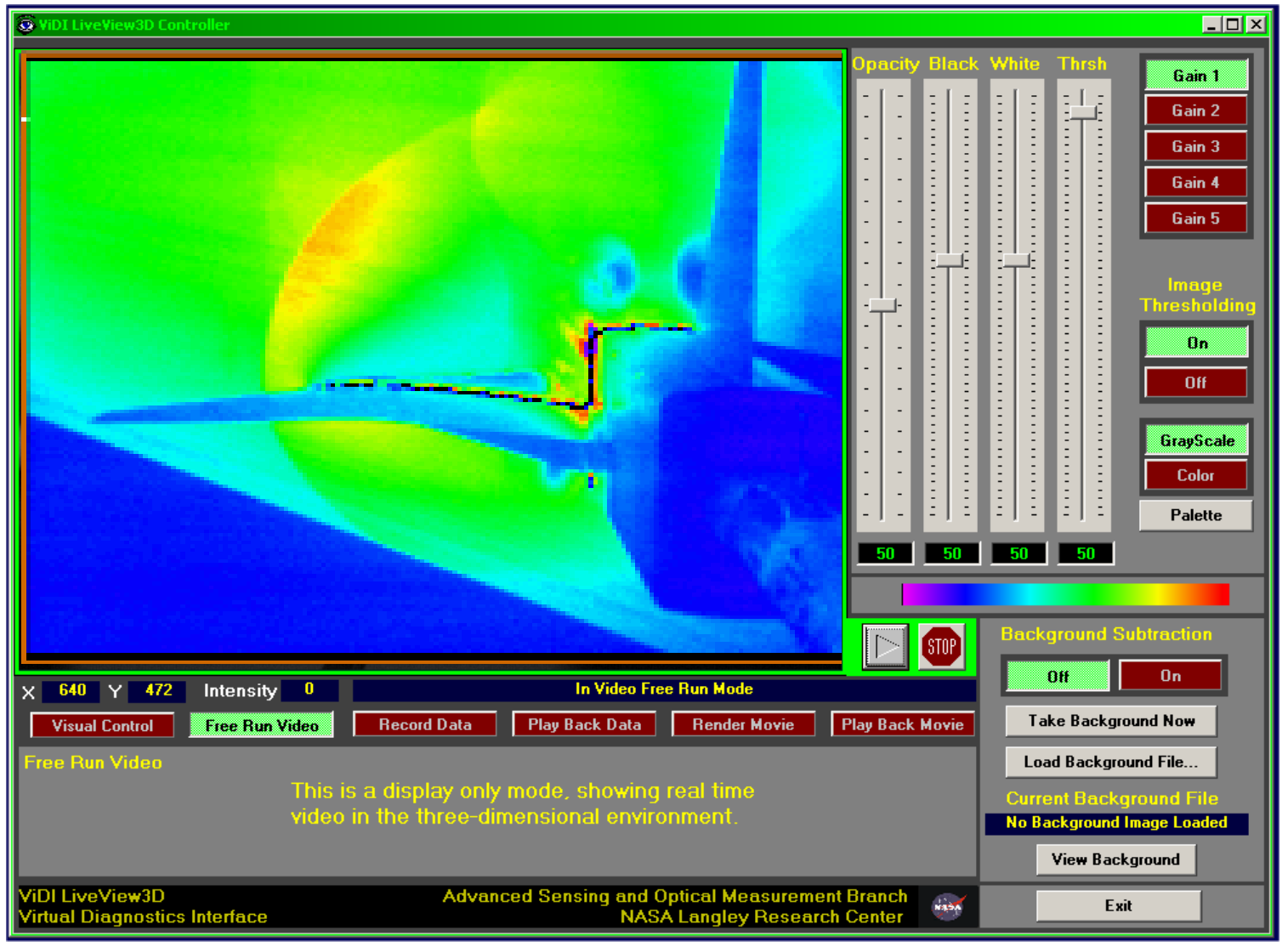

Figure 1. User interface for stand alone LiveView3D LiveVideo program. The interface controls the frame grabber hardware and real time image processing. This version of the software is used when video data is being displayed in the virtual environment without a connection to a DAS. The version of LiveView3D that is fully integrated with the DAS contains the same interface and functionality. 


\section{Laser Light Sheet Flow Visualization}

Laser light sheet flow visualization is one of the simplest and most widely used optical diagnostic techniques for wind tunnel testing. This technique allows a researcher to qualitatively visualize off-body flow structure for identification of prominent flow features, such as the location of vortices, shear layers, shocks, etc. Laser light sheet flow visualization is accomplished by forming the output beam of a moderate-power continuous wave laser into a thin, planar sheet of laser light. The sheet of laser light is then directed into the wind tunnel test section and aligned to illuminate the region-of-interest. Smoke or other particulate seeding is introduced into the flow upstream of the test section, which follows the flow streamlines as it convects downstream. The smoke particles ultimately pass through the light sheet and scatter the laser light, highlighting prominent flow features. The resulting flow visualization patterns are typically observed and recorded using a video camera and recording equipment.

\section{a. Supersonic Flow Visualization over a fighter configuration.}

The NASA LaRC UPWT is exceptionally well-suited for flow visualization. The UPWT is a closed-loop, variable density, continuously running supersonic wind tunnel with two independent 4-x 4-x 7-foot test sections $^{7}$. One test section operates at speeds from Mach 1.5 to 2.9, while the second test section operates at speeds form Mach 2.3 to 4.6. Laser light sheet flow visualization is used often in the facility to investigate off-body flow features about the test article. Flow seeding is accomplished by injecting a small amount of liquid water into the tunnel while operating. The water evaporates in the low density flow and re-condenses when accelerated to supersonic speeds. This produces a uniform "fog" throughout the test section that is ideal for laser light sheet flow visualization.

One of the first applications of LiveView3D in the UPWT was to use laser light sheet flow visualization to investigate the shock and vortical flow structures about a generic fighter configuration tested at supersonic speeds. The experimental setup is shown in Figure 2. A 5-watt argon-ion laser and light sheet forming optics were used to illuminate a planar region about the model for flow visualization. A video camera mounted in the model sting was used to visualize the flow patterns. The resulting field-of-view was approximately $30 \mathrm{~cm}$ square. The model was traversed longitudinally through the laser sheet to visualize the flow at different streamwise stations along the model.

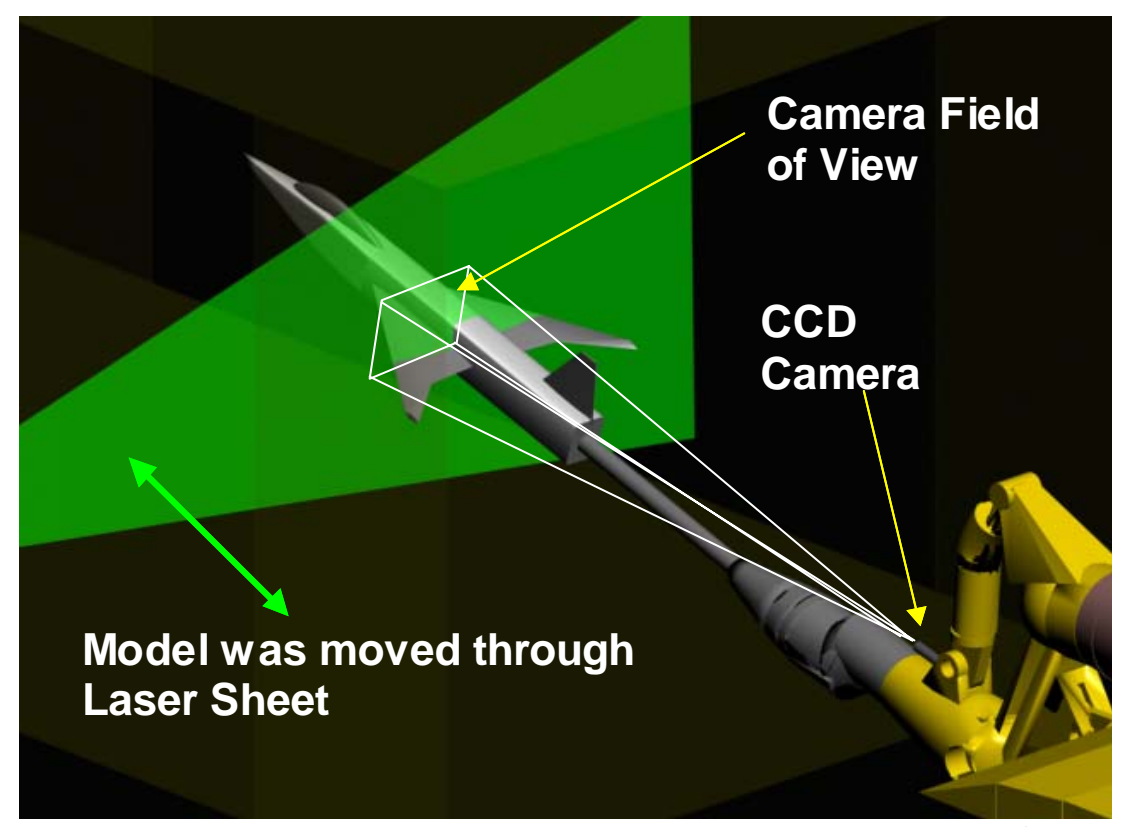

Figure 2. Laser light sheet experiential setup used for LiveView3D in the NASA Langley Unitary Plan Wind Tunnel 
Setting up the LiveView3D system for optimal use involved the following steps: spatial registration of the visualization plane in the virtual environment, scaling the size of the video imagery to proper size within the virtual environment, and obtaining background images. The spatial location of the light sheet plane in the test section was determined prior to conducting the test. This plane was spatially registered within the LiveView3D virtual environment by measuring the distance from a reference point (in this case the nose) and creating nodes to which this plane would be moved to. Image scaling was accomplished by acquiring video of the model and sizing the features of the video image to match the virtual model. Prominent points such as wing tips and the wing-body juncture made it easy to align the video precisely. Images of graduated scales were also taken, and while they worked well, were deemed unnecessary in lieu of alignment with the model features. Background images were acquired and stored while the facility was operating and with the laser on, but no seeding injected into the flow. Acquiring background images in this manner ensured that the background images contained light from all extraneous sources, including laser flare from the model surface in its aerodynamically loaded position. An average background image was acquired and stored for each tunnel and model condition to be tested. This completed the initial setup phase of operation for LiveView3D.

For this test entry, a pre-existing CAD model of the facility (UPWT) existed, and a three-dimensional model of the test configuration was provided by the party responsible for building the model. Thus, preparation of the virtual environment took about two hours. This can often be done well in advance of the test. Experience has shown that acquiring the background video and finalizing LiveView3D to display the data approximately requires an addition two hours. This work is usually accomplished as other wind tunnel preparation work is being conducted, so LiveView3D has generally had a minimal impact on the facility operational schedule.

The LiveView3D system was ready for use after the initial setup. Flow seeding was initiated after acquiring the background images, and the video produced by the flow visualization camera was streamed into the virtual environment. The background image corresponding to the tunnel / model condition being investigated was subtracted from the incoming video stream to remove the effects of extraneous light and laser flare, and additional gain was applied using software controls to optimize video contrast. A false color palette was applied to accentuate the flow structure, and the resulting image was mapped into the virtual environment for display. The computations required to perform these steps reduced the frame update rate in the virtual environment to approximately 12 to $15 \mathrm{~Hz}$, which was adequate to maintain a sense of continuity in the video stream.

The LiveView3D system allows the user to navigate through the virtual environment as the data is being displayed. The scene can be viewed from any angle, with any level of magnification (figure 3 ). The user may also choose to incorporate the facility in the visualization, make the facility semi-transparent (to act as a reference without overpowering the data visualization), or be completely hidden. Experience has shown that through experimentation with the settings, LiveView3D affords a wide variety of flexibility to enhance and optimize the information contained within the flow visualization. 


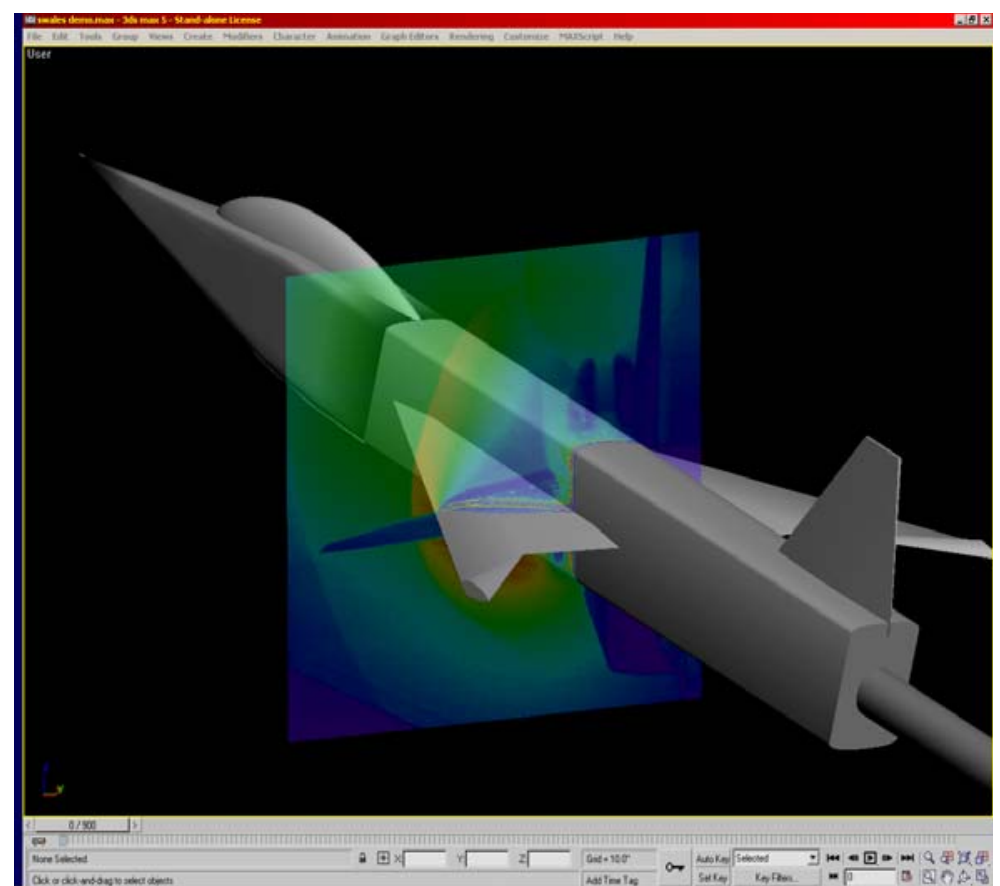

Figure 3. Example of interactive three-dimensional laser light sheet visualization in the virtual environment. This data has undergone background subtraction and false color imaging.

As the video is being streamed through LiveView3D and into the virtual environment, the user may choose to save the frame grabbed images to hard drive. Each captured image is saved in a non-compressed bitmap format, assigned a filename containing a numerical sequence. These data files may be recalled and substitute the live video stream, for playback in the interactive virtual environment. This data may also be used to render a stand-alone movie file in an industry standard movie file format (figure 4). Software for movie playback is available for all computer platforms.

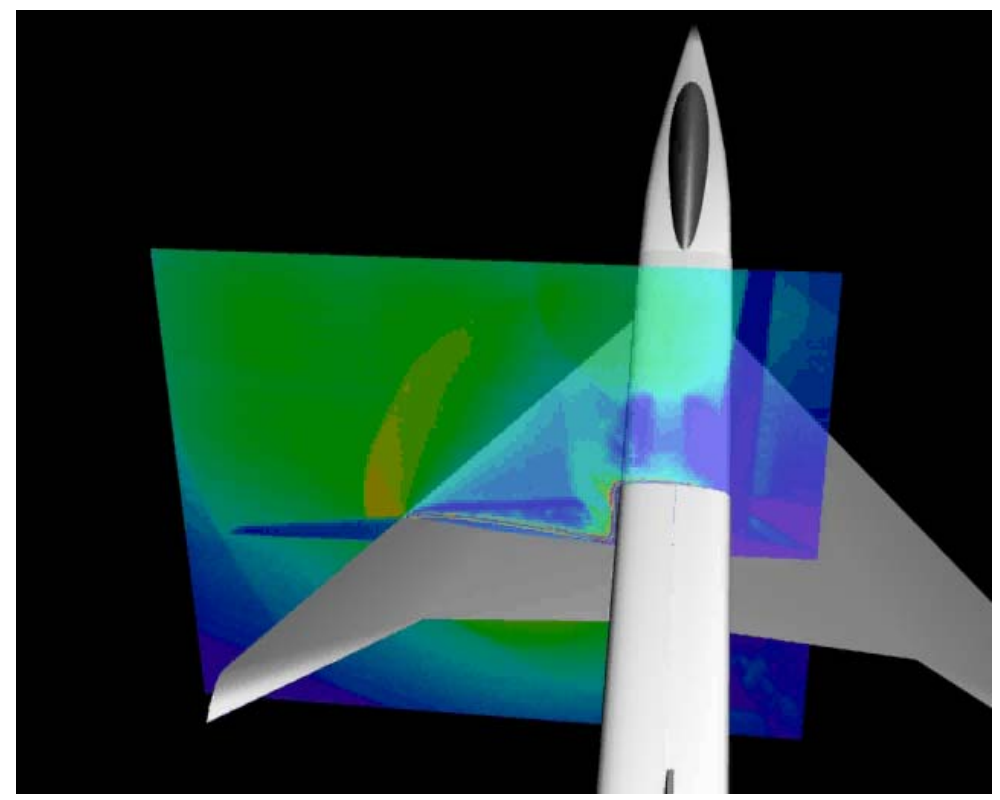

Figure 4. Rendered image of data laser light sheet flow visualization. Note that the transparency setting was used to allow the user to see through the data, which helps to orient the user to the data plane. 


\section{b. Subsonic Flow visualization on a race car configuration}

In January 2006, Old Dominion University (ODU) conducted a test utilizing a NASCAR-class race car in the NASA LaRC 14-x 22-Foot Subsonic Wind Tunnel. The primary purpose of the test was to evaluate an automotive force balance system developed by ODU. A secondary objective included collaboration with NASA to evaluate the applicability of several different advanced instrumentation systems for race car testing. LiveView3D was one of the systems evaluated, used in conjunction with laser light sheet flow visualization to visualize the separated flow region over the rear window, deck, and spoiler of the car.

The 14-x 22-Foot Subsonic Wind Tunnel can be run as an open jet or as a closed wall facility. For this experiment, the wind tunnel was configured as an open jet, with walls and ceiling raised above the flow. A 10 -Watt $\mathrm{Nd}: \mathrm{YVO}_{4}$ laser emitting at $532 \mathrm{~nm}$ was placed above the wind tunnel ceiling, approximately 25 feet above the region-of-interest. A pair of galvanometer scanning mirrors were utilized to create the light sheet. Custom electronics allowed the mirrors to be remotely controlled such that the laser light sheet position and extent could be adjusted during tunnel operations. The laser light sheet was projected through a glass window in the tunnel ceiling and down towards the car to illuminate the region-of-interest (figure 5). Flow seeding was accomplished by using a mineral oil smoke generator placed in the wind tunnel settling chamber.

A standard RS-170 monochrome video camera was placed outside of the flow perpendicular to the center of the laser light sheet to minimize perspective distortions. The video was fed directly to the LiveView3D computer. Background image subtraction was also utilized in this case in order to remove undesirable effects caused by extraneous light. Once the video image was scaled to the virtual environment the flow visualization could be analyzed in context to the model geometry (figure 6).

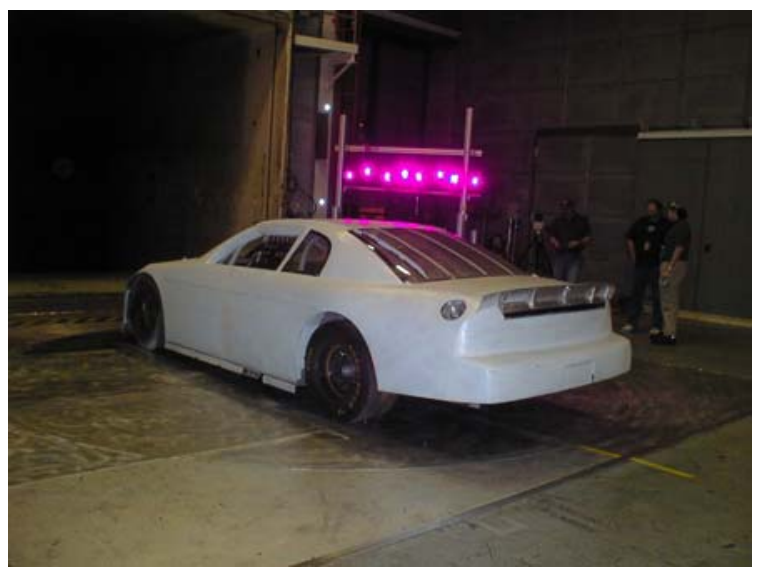

a.

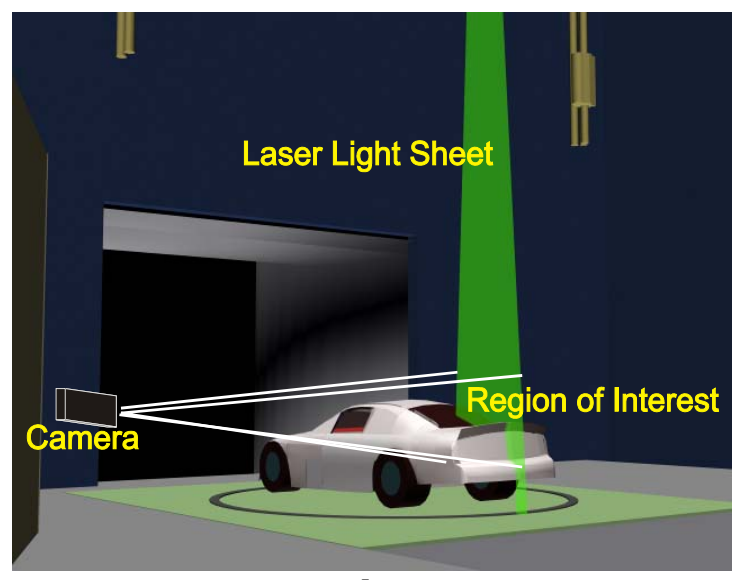

b.

Figure 5. (a)Photograph of car in the 14-x 22-Foot Subsonic Wind Tunnel, (b) Rendition of laser light sheet and camera setup for LiveView3D data visualization. 


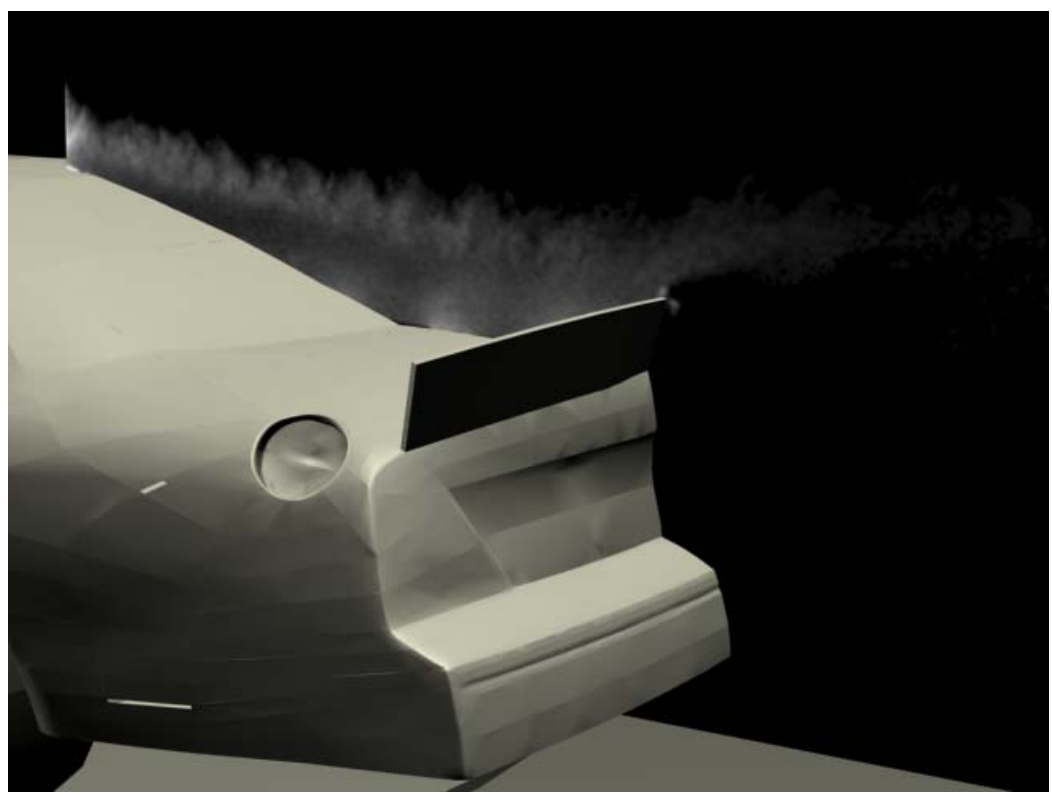

Figure 6. LiveView3D rendering of laser light sheet flow visualization over the rear deck of the stock car configuration.

\section{B. Schlieren Flow Visualization}

Schlieren flow visualization is a well-established and commonly used method for visualizing density gradients in flows. The LaRC UPWT consistently uses schlieren visualization to view shock patterns around models (figure 7). A video camera transmits the schlieren imagery to the data acquisition room, where it has been traditionally viewed on large televisions. Using LiveView3D, the schlieren can be placed in the virtual environment in real time.

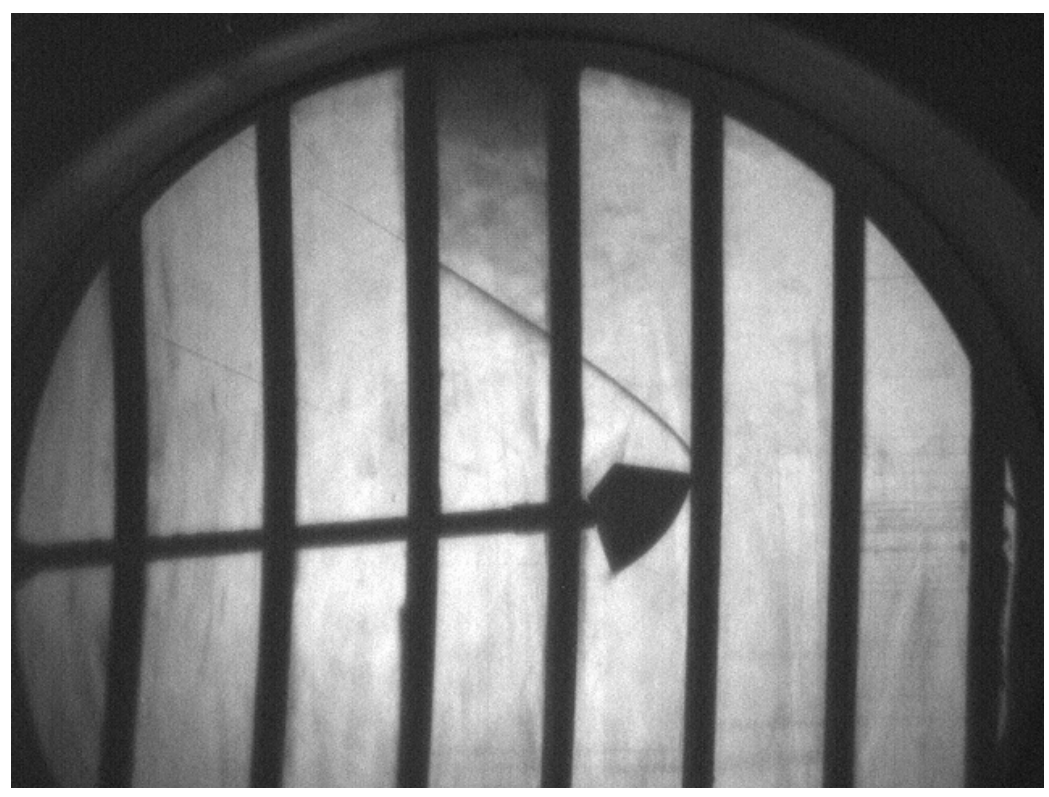

Figure 7. Schlieren image of the supersonic flow field about a model of the NASA Crew Exploration Vehicle in the LaRC UPWT. Vertical bars are slats that hold the test section windows in place. 
Currently a RS-170 video camera is used in the UPWT schlieren system to image the flow density gradients. Thus this video signal can be directly connected to the frame grabber used by the LiveView3D system. The video image is captured and processed as described above for laser light sheet flow visualization. For schlieren imagery, however, background image subtraction is not performed. This is because sufficient image quality was obtained without background subtraction, deeming it unnecessary to spend the additional tunnel occupancy time required to obtain background images for each tunnel / model condition tested. Since the schlieren image actually represents the density gradient integrated across the test section, the data is arbitrarily depicted on the model centerline for ease of viewing. Representative results are shown in figure 8 .
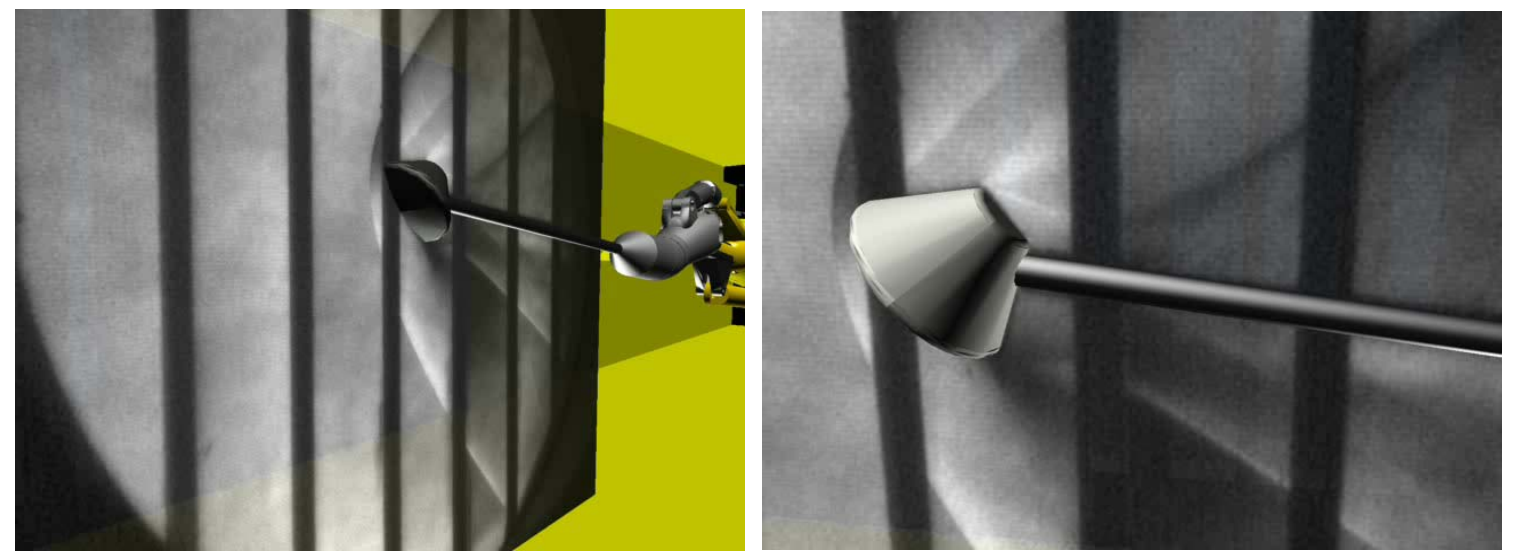

Figure 8. LiveView3D renderings of the schlieren imagery about of the CEV model in the LaRC UPWT. Imagery such as this was available in real time during the test.

\section{Utilizing the Wind Tunnel DAS System.}

Wind tunnel DASs are designed to capture, manipulate and record a variety of parameters pertaining to pressures, forces, temperatures and other environmental conditions. With LiveView3D, this information can be converted into an easy to interpret visualization of the state of the experiment in real time. LiveView3D has been integrated with the DAS utilized by the LaRC UPWT. This has provided the ability to accept up to five hundred channels of scalar information on tunnel status and data values processed to engineering units or dimensionless coefficients.

Communications with the DAS was established via an Ethernet connection utilizing the TCP/IP communications protocol. To organize the flow of information, the DAS data was divided into two categories; test status information and test data. Test status information such as the current test, run and point, Mach and Reynolds numbers are displayed prominently on screen as well as being used to organize the test data. The test data pertains to the instrumentation measurements, and were assigned to attributes of individual three-dimensional objects in the virtual environment (figure 9). 


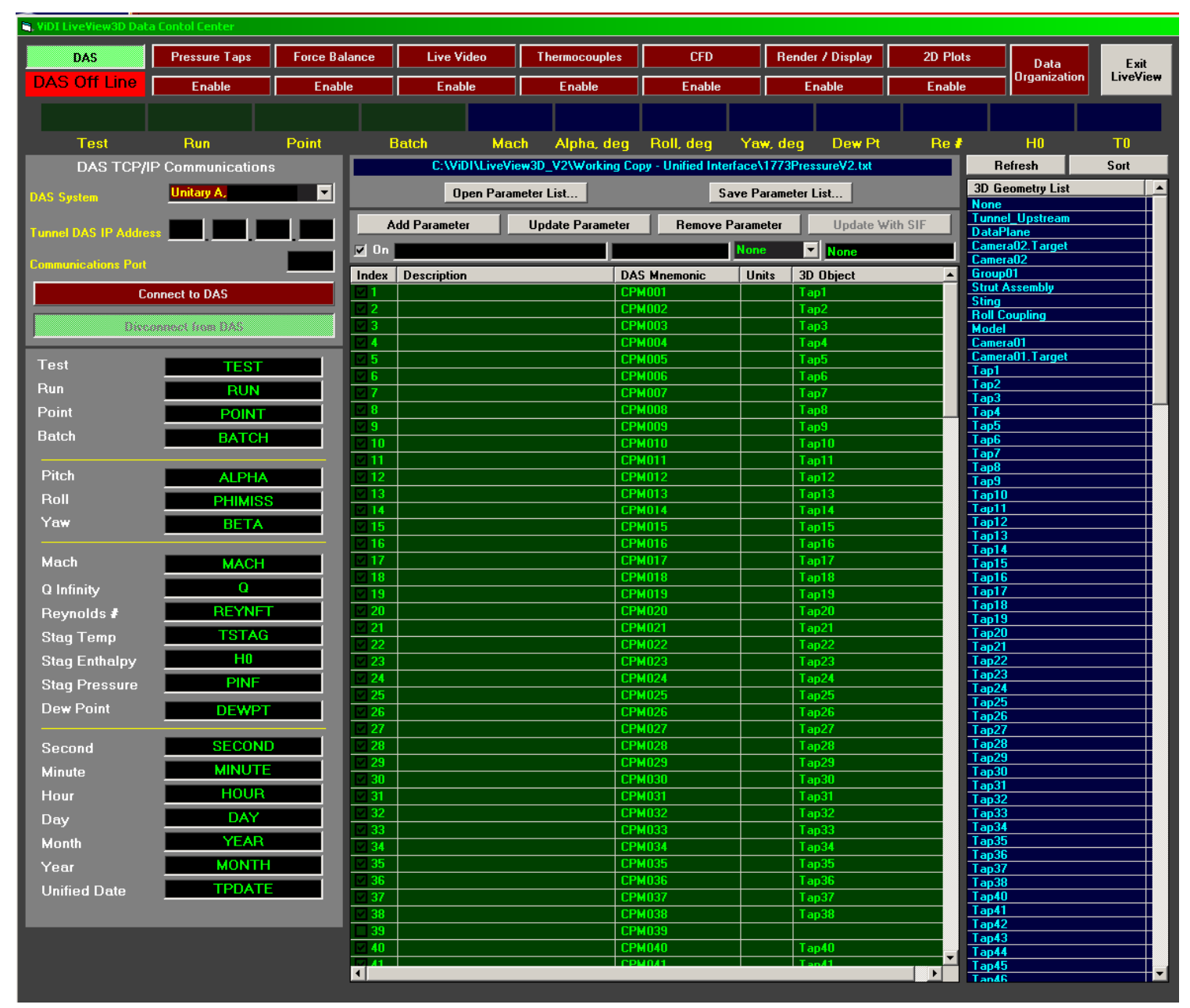

Figure 9. LiveView3D DAS communications user interface. The user selects DAS parameters that are to be received by LiveView3D, as well as assigning DAS parameters to three-dimensional objects such a pressure taps in the virtual world. Test status information parameters are shown on the left while test data values are listed in the center. The column on the right lists all of the threedimensional objects in the virtual environment.

The LiveView3D program was designed to poll the DAS computer at a $2 \mathrm{~Hz}$ rate, looking for changes to the test, run, point or batch number. When a change occurred, LiveView3D initiated a transfer of the desired DAS data parameters. These values are immediately received and parsed into appropriate internal data structures. LiveView3D then saved the values to hard drive and proceeded to update the graphics by commanding a redraw of elements of the virtual environment.

\section{A. Auto-Tracking - Matching Test Configuration}

The Auto-Tracking feature within LiveView3D allows the roll, pitch and yaw of the virtual model to mimic the real world configuration. The three-dimensional virtual geometry of the sting mechanism has been carefully designed to simulate the mechanical linkages of the actual sting. Thus, when the DAS records the roll, pitch and yaw of the model, the values are passed to the appropriate sting components (figure 10). By enabling the Auto-Tracking feature, LiveView3D automatically adjusts the configuration every time a data point is acquired. This feature eliminates the need for an operator to manually reposition the virtual test article to match the actual experimental condition, thus greatly reducing operator workload during rapid data acquisition. 

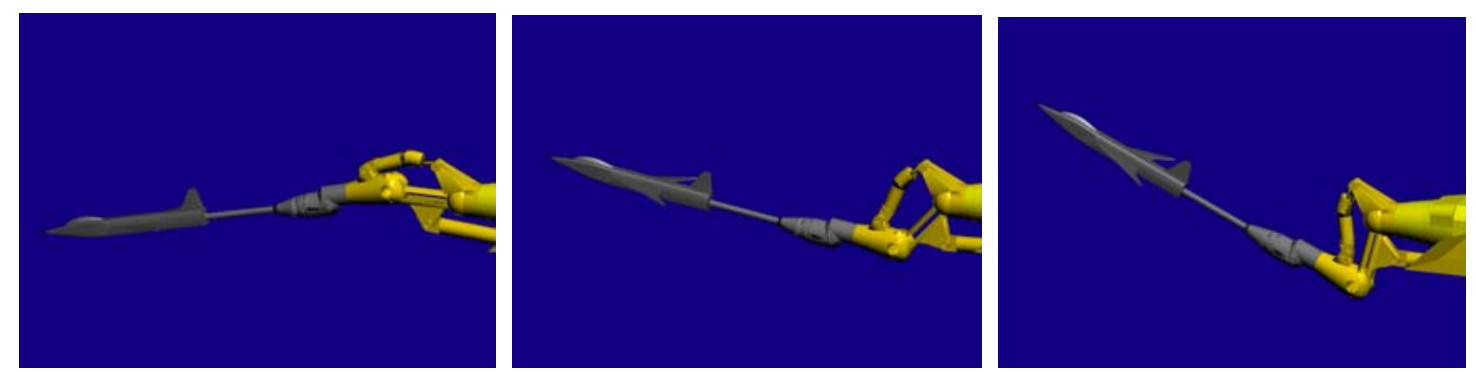

Figure 10. Sequence depicting angle of attack change controlled automatically via DAS pitch data.

\section{B. Force Balance Data - Using a reference axis system}

A force balance is a precision instrument used to determine aerodynamic loads during a test. Six component force balances measure three orthogonal forces and moments about each of the corresponding axes. The DAS acquires this data and computes values in engineering units and dimensionless coefficients in real time. A recent test utilizing a force balance in the UPWT involved a NASA Crew Exploration Vehicle (CEV) capsule, a manned spacecraft similar in configuration but larger than an Apollo capsule.

The LiveView3D software was configured to receive dimensionless coefficients of the force balance data from the DAS. The depiction of the data consists of an orthogonal three-dimensional axis reference system with each axis made up of a thin cylinder of differing color (figure 11). The length of a thicker cylinder placed collinearly along each reference axis represented the magnitude of the force coefficient. Likewise, a series of three small radius hoops surrounds the force axis reference. Each of the orthogonally aligned hoops represents a moment about the corresponding axis, and a larger radius arc section collocated along each reference hoop represents the moment of the magnitude of the coefficient of the moment.

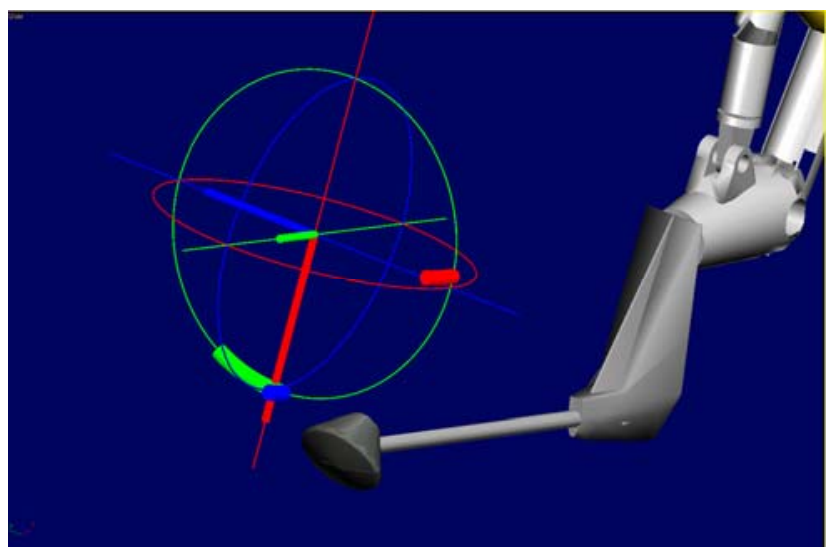

Figure 11. Graphical depiction of forces and moments in the virtual environment. Thicker bars aligned with the axis represent forces, while the arced bars depict the moment about each of the corresponding axis. These graphical elements are linked to roll, pitch and yaw in the appropriate axis.

\section{Surface Pressure Taps}

One of the most commonly employed wind tunnel measurement techniques are surface pressure taps. These small orifices provide a passageway for air to pass from the surface of the model to instrumentation designed to measure the pressure. The DAS system is capable of accepting data from hundreds of individual pressure taps, and can compute pressures in engineering units or dimensionless coefficients in real time. Using LiveView3D, the data from each pressure tap can be place in-situ in the virtual environment so the pressure distribution across the entire instrumented area can be analyzed in a single glance. 
In order to use LiveView3D to analyze surface pressure tap data, the user has to create a small cylinder to represent the pressure at a given tap location. Creation of the cylinder is done automatically within the LiveView3D software when the user specifies the coordinates of a pressure tap. The cylinder is "linked" to the three-dimensional geometry of the test article, so as the test article translates or rotates the position of the cylinder will remain constant relative to the test article. The cylinder is then assigned to the appropriate channel of DAS data such that the information from the actual pressure tap will correlate with the pressure tap representation in the virtual environment. This channel ties back to either a coefficient of pressure or pressure measurement in engineering units.

Once the taps are assigned to the proper DAS channels, the user may specify which taps are actively displaying data at any given time. The taps can be organized into multiple sets of groups, which each group containing taps that are in a logical set, such as a row along a chord on a wing, or the centerline of a fuselage. A graphical user interface showing three two-dimensional projections of the taps as located in three-dimensional space in the virtual model allows the user to easily group the taps (figure 12). Grouping of taps is also important for data plotting, which will be described below.

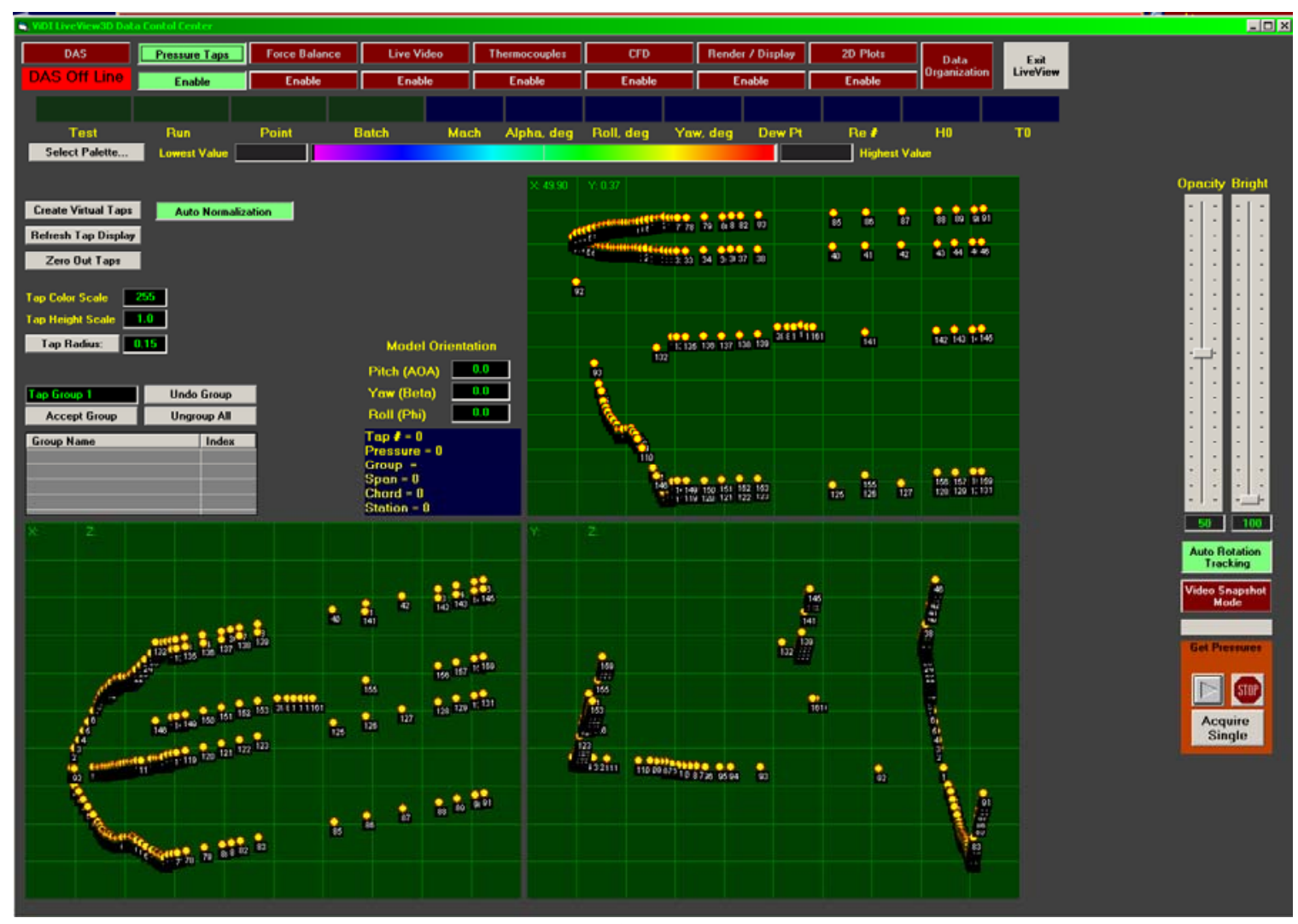

Figure 12. LiveView3D pressure tap user interface. Each pressure tap on the model is depicted as a yellow circle in the three two-dimensional projections of the virtual environment. Spatial coordinates for each tap are read directly from the virtual environment and mapped to scale. The user may select any number of taps to be in a group, and any number of groups can be created. To display the data in the virtual environment, the user has to enable a group.

When pressure data is received from the DAS, LiveView3D parses the information and pairs the incoming data with the virtual taps. Once this process is complete, the programs will proceed to update the virtual environment. The tap data is normalized to a reference value, and a color value is assigned from a 256 value look-up-table based on scaling between specified minimum and maximum pressure values. The length of the cylinder is determined by multiplying the pressure data by a gain value and using that value to set the length of the cylinder. The example shown in figure 13 is again the NASA CEV capsule, tested in 
the Langley UPWT. LiveView3D is capable of updating the graphics in this scene within one second of receiving the DAS data.

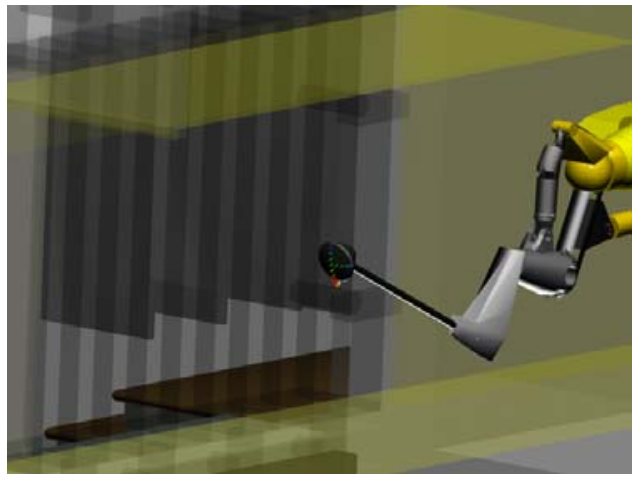

a.

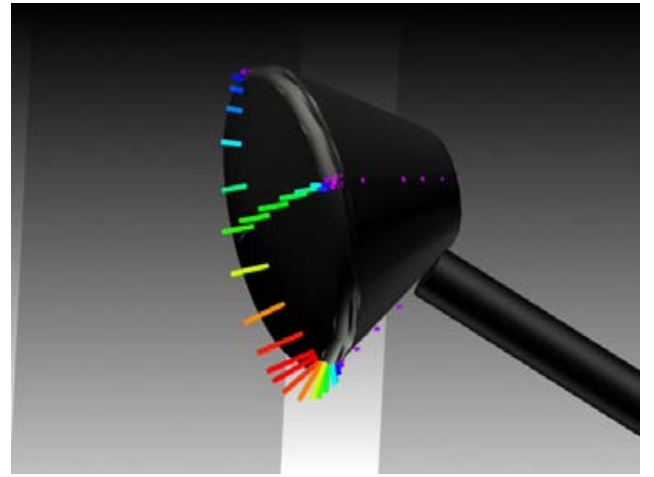

b.

Figure 13. NASA CEV pressure tap data displayed in the virtual environment. (a.) depicts the capsule with a cut-away of the facility to help put the data set in context with the test conditions. (b.) is a close-up of the pressure distribution. Such imagery is available to the user in real time.

\section{Two-Dimensional plotting}

The interactive virtual environment is a new and unique tool for displaying data, but it is best used as a qualitative analysis tool, where trends and global patterns are easily discernable. For detailed qualitative data analysis, a traditional two-dimensional plot of the data remains an integral form of data presentation. As such, LiveView3D incorporates a plotting capability designed to work in unison with the virtual environment (figure 14).

The user may choose to plot a wide variety of data. Any of the three forces or moments can be plotted against roll, pitch or yaw, and displayed as a function of time, or groups of pressure taps vs. location can be plotted. An auto-scaling feature creates a plot designed to encompass all of the data being presented.

However, the user has a wide range of controls to override the automation in order to better format plots for given sets of conditions. 


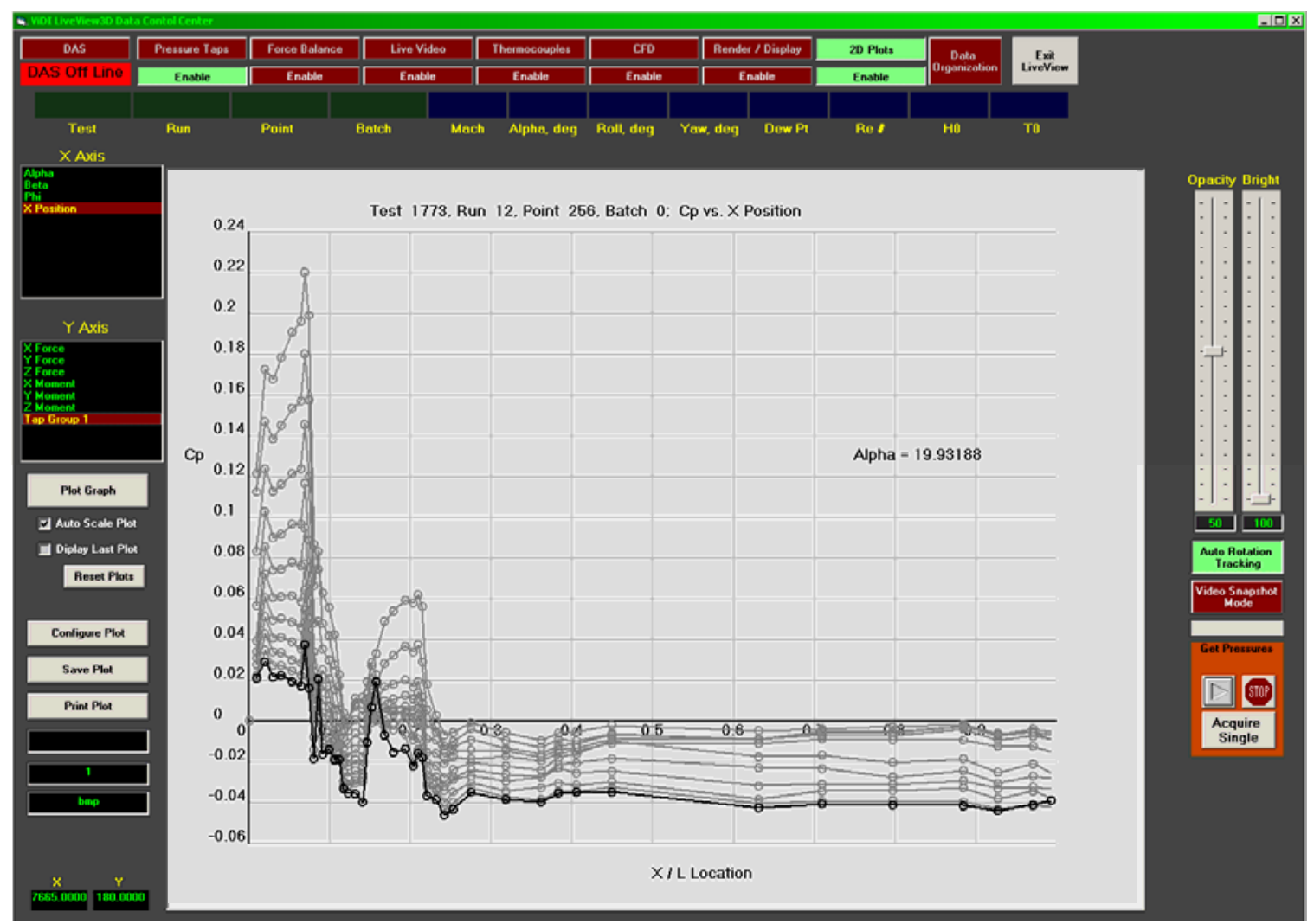

Figure 14. Two-Dimensional plotting capability within LiveView3D. This display may be updated in real time along with the three-dimensional virtual environment. Multiple data lines trace pressure variation as a function of angle of attack in this example.

\section{E. Automated operation and saved output.}

One of the main goals of the LiveView3D system is that its operation not be so complex that the researchers are unable to spend time analyzing the data in real time. To this end, most of the operations of LiveView3D are automated. This allows the user to interact with the three-dimensional virtual environment instead of spending time routing data to the correct locations. LiveView3D polls the DAS and updates as warranted on a continuous basis. The system will record all of the data it receives onto the hard drive of the LiveView3D computer. It also can run in a "Video Snapshot Mode", allowing it to record a single frame of video as soon as it detects data has been acquired by the DAS. A log file keeps track of each point that has been acquired, and any corresponding video images saved to disk.

In addition to saving the individual data elements to hard drive (which allows the user to play it back later in the virtual environment), a high quality rendering of the virtual environment, overlaid with pertinent tests information, can be created and saved (figure 15). Each rendering requires several seconds to create, and is saved to hard drive with a file name corresponding to the test, run and point numbers of the given test condition. Also, if a two-dimensional plot is being created, it too is saved as a bitmap with a corresponding file name matching the rendered image file (figure 16). 


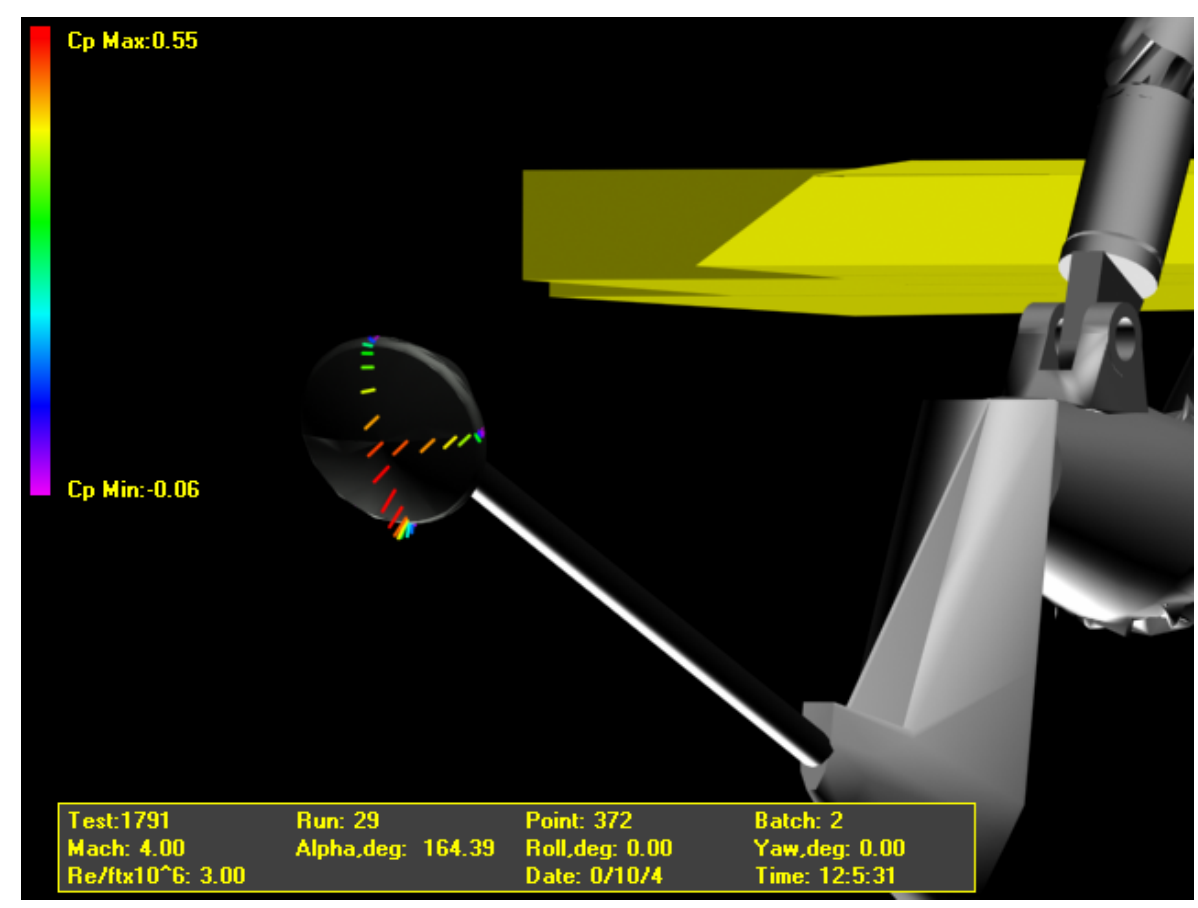

Figure 15. A rendering of the NASA CEV created in real time. This bitmap has test status information overlaid on it. The user can choose to have a bitmap is saved for each data point acquired.

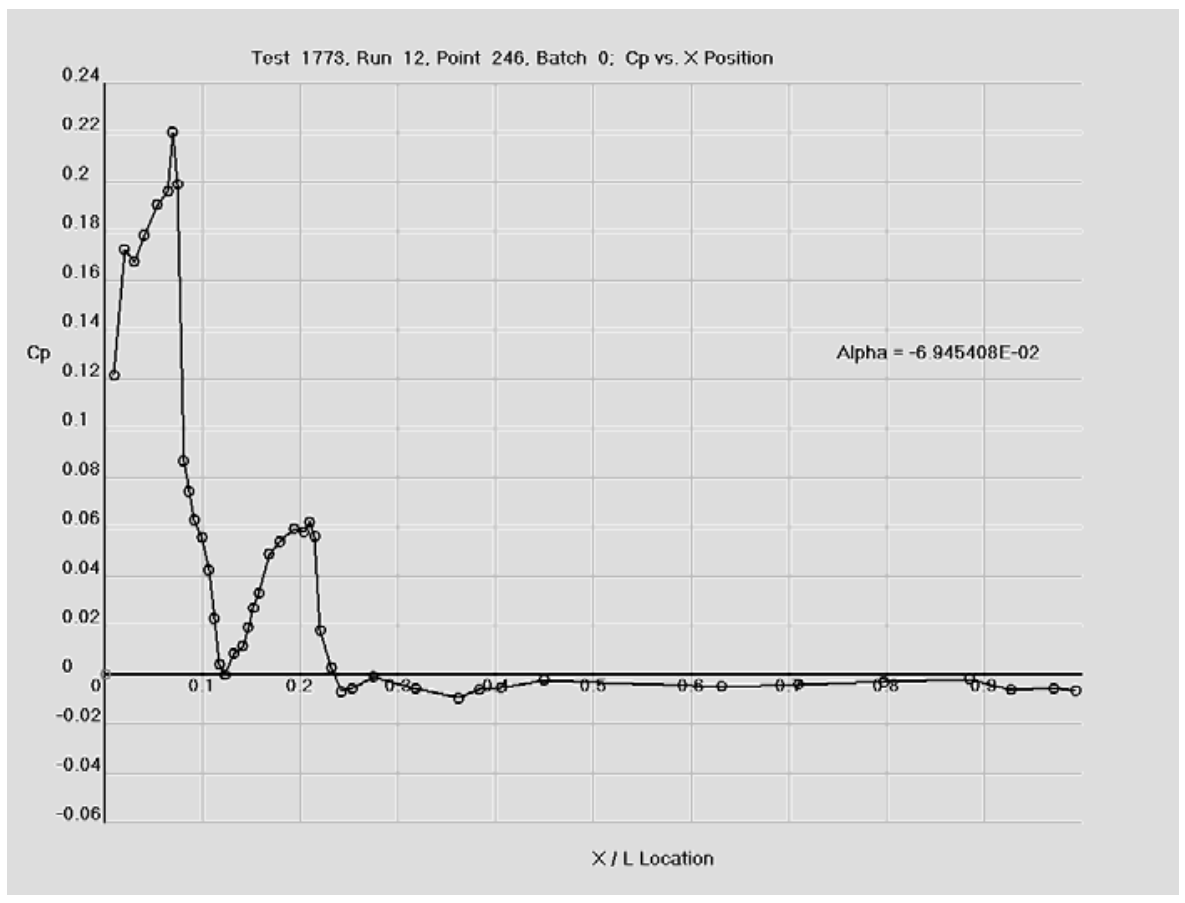

Figure 16. A two dimensional data plot can be created and saved in real time as a bitmap file. Filenames are based on Test, Run and Point values from the DAS, and correspond to the filenames used for the renderings shown in Figure 15 as well as data files saved to the LiveView3D computer containing the original data. 


\section{Future Work}

There are several avenues of development that will be taken in the future. Among them is the ability to incorporate image warping in real time for the video stream. Image warping will provide the ability to remove optical aberrations and camera perspective distortion through the use of a spatial calibration taken just prior to the test. In addition, enhancements to the data plotting routines and utilities to more fully automate the setup of the virtual environment, such as creating the pressure tap cylinders, will be implemented.

The primary focus of future work will reside with visualizing and comparing computational fluid dynamics (CFD) and finite element modeling (FEM) results along with the experimental data, both in real time and in post-test data visualization. Routines for importing a variety of computational formats will be incorporated in LiveView3D, as will tools designed for detailed comparisons of the data ${ }^{8}$. As experience is gained, the program will be fine-tuned, with development continuing in a modular fashion. This will allow extended capabilities and applications in new facilities to be optimally incorporated.

\section{Acknowledgments}

The authors would like to thank John Micol, Ricky Hall, and Dan Cole at the NASA Langley Unitary Plan Wind Tunnel for providing unwavering support and broad access to the wind tunnel and data acquisition system. In addition, the efforts of Dr. Drew Landman, Eric Koster and the entire Langley Full Scale Wind Tunnel crew of Old Dominion University for incorporating LiveView3D with the race car testing was highly appreciated.

\section{References}

${ }^{1}$ Wright, K.D., Fleming, G.A., “Advanced Wind Tunnel Measurement Technologies at NASA Langley Research Center”, First Joint Meeting of the Supersonic Tunnel Association International (STAI) and Subsonic Aerodynamic Testing Association (SATA) Meeting, Buffalo, NY, May 15-19, 2005

${ }^{2}$ Windows is a registered trademark of Microsoft Corporation in the United States and other countries.

${ }^{3}$ Microsoft Visual Basic a registered trademark of Microsoft Corporation in the United States and other countries

4 3ds Max a registered trademark of Autodesk Inc.

${ }^{5}$ ActiveX is a registered trademark of Microsoft Corporation in the United States and other countries

${ }^{6}$ Schwartz, R.J., Fleming, G.A.,'LiveView3D: Real Time Data Visualization for the Aerospace Testing Environment”, AIAA-2006-1388, 44 ${ }^{\text {th }}$ AIAA Aerospace Sciences Meeting and Exhibit, Reno, Nevada, Jan. 9-12, 2006.

${ }^{7}$ Erickson, G.E., "Overview of Selected Measurement Techniques for External Aerodynamic Testing in a Supersonic Wind Tunnel Facility”, AIAA 2000-2396, AIAA Fluids 2000 Conference, 19-22 June, 2000, Denver CO.

${ }^{8}$ Schwartz, R.J., "ViDI: Virtual Diagnostics Interface Volume 2-Unified File Format and Web Services as Applied to Seamless Data Transfer", NASA/CR-2003-212668, December 2003 